\title{
Identification of Thrust, Lift, and Drag for Deep-stall Flight Data of a Fixed-wing Unmanned Aircraft
}

\author{
Torbjørn Cunis, ${ }^{1}$ Tobias Leth,${ }^{2}$ Luminita C. Totu, ${ }^{3}$ and Anders la Cour-Harbo ${ }^{4}$
}

\begin{abstract}
In this paper, we consider a small unmanned aircraft and data collected during regular and deep-stall flight. We present an identification method for the thrust force generated by the propulsion system based on the in-flight measurements where we make use of the well-known linear and quadratic approximations of the lift and drag coefficients, respectively, for low angles of attack. This overcomes the lack of propeller thrust measurements and the obtained models are successfully evaluated against CFD simulation. The identified thrust model proves applicable beyond low angles of attack, thus enabling force estimation in the full flight envelope.
\end{abstract}

\section{INTRODUCTION}

A particular feature of small unmanned aircrafts (UAs) is the absence of landing gear. Promoting a lightweight construction, it complicates landing. While some existing drones simply "crash land" and separate [1] or glide after impact [2], another technique exists. By deliberately entering a deep stall, a drone lands by a vertical descend and stays at the point of impact. [3, 4] This is termed deep stall landing, and one such UA is the Cumulus One.

To land on its belly and lest to shatter upon impact, Cumulus maintains a pitch around zero degrees during vertical descent. This requires controlled flight at high angles of attack, exceeding the stall point. Various offthe-envelope control approaches have been developed [4-6], commonly approaches requiring accurate models of the aerodynamics (see also [7] and references herein). In $[8,9]$, the aerodynamics of small UAs were modeled based on their geometry; and accurate measurement technologies have boosted the in-flight estimation of aerodynamic coefficients [10-12]: the latter involves the computation of forces during flight based on trajectories, which are usually recorded using optical tracking technology. In order to neglect the thrust force in these equations, studies

\footnotetext{
${ }^{1}$ Torbjørn Cunis is with the Department of Information Processing and Systems, ONERA - The French Aerospace Lab, 31055 Toulouse, France; torbjoern.cunis@onera.fr and the Drones Research Group, French Civil Aviation School, 31055 Toulouse, France.

${ }^{2}$ Tobias Leth is with the Department of Electrical Engineering, Automation \& Control, University of Aalborg, 9220 Aalborg East, Denmark. tol@es.aau.dk

${ }^{3}$ Luminita C. Totu is with the Department of Electrical Engineering, Automation \& Control, University of Aalborg, 9220 Aalborg East, Denmark. Ict@es.aau.dk

${ }^{4}$ Anders la Cour-Harbo is with the Department of Electrical Engineering, Automation \& Control, University of Aalborg, 9220 Aalborg East, Denmark. alc@es.aau.dk
}

were conducted for gliders or with the thrust simulated based on the propeller shape [11]. Yet, identification of the thrust provided by the vehicles' propellers is a separate task [13-15]. A few studies have been conducted into thrust force estimation of marine propellers [16] and aeronautical turbofan engines [17]. However, even they required prior knowledge of the propulsion systems. Instead, we will employ well-known approximations of lift and drag forces at low angle of attack to separate the estimated aerodynamic and thrust forces and identify a thrust model using linear least-squares optimization [18].

This paper presents data from a flight of the $\mathrm{Cu}-$ mulus UA, where deep stall landings are initiated but aborted. The recorded flight data includes high angles of attack, which yields an experimental validation of the full-envelope aerodynamic coefficients. As there is no propulsion model for the Cumulus propeller during flight, we identify the applied thrust based on the flight data for low angles of attack and extend the model to the full envelope. Accurate drag and lift models over the full flight envelope will allow us to derive control strategies for precision descents and landing on moving targets.

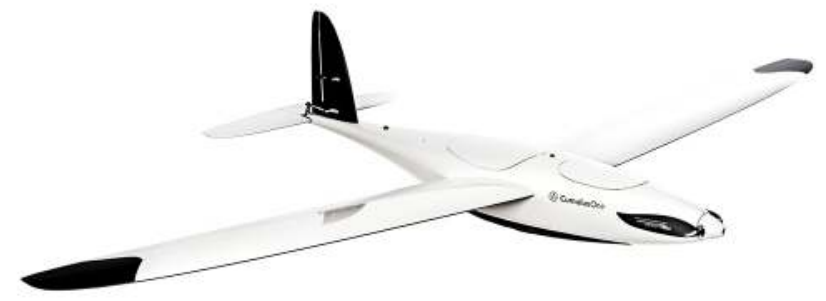

(a) Digital rendering of Cumulus One ready for flight. [19]

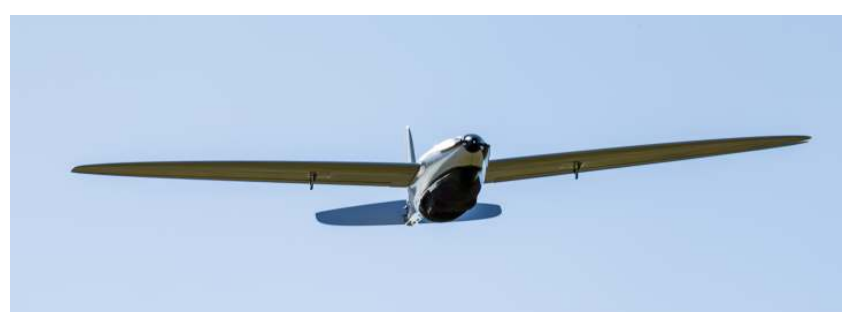

(b) Photograph of Cumulus One in flight. [19]

Fig. 1: The Cumulus One UA. Note how the propeller blades are folded back when not spinning. 


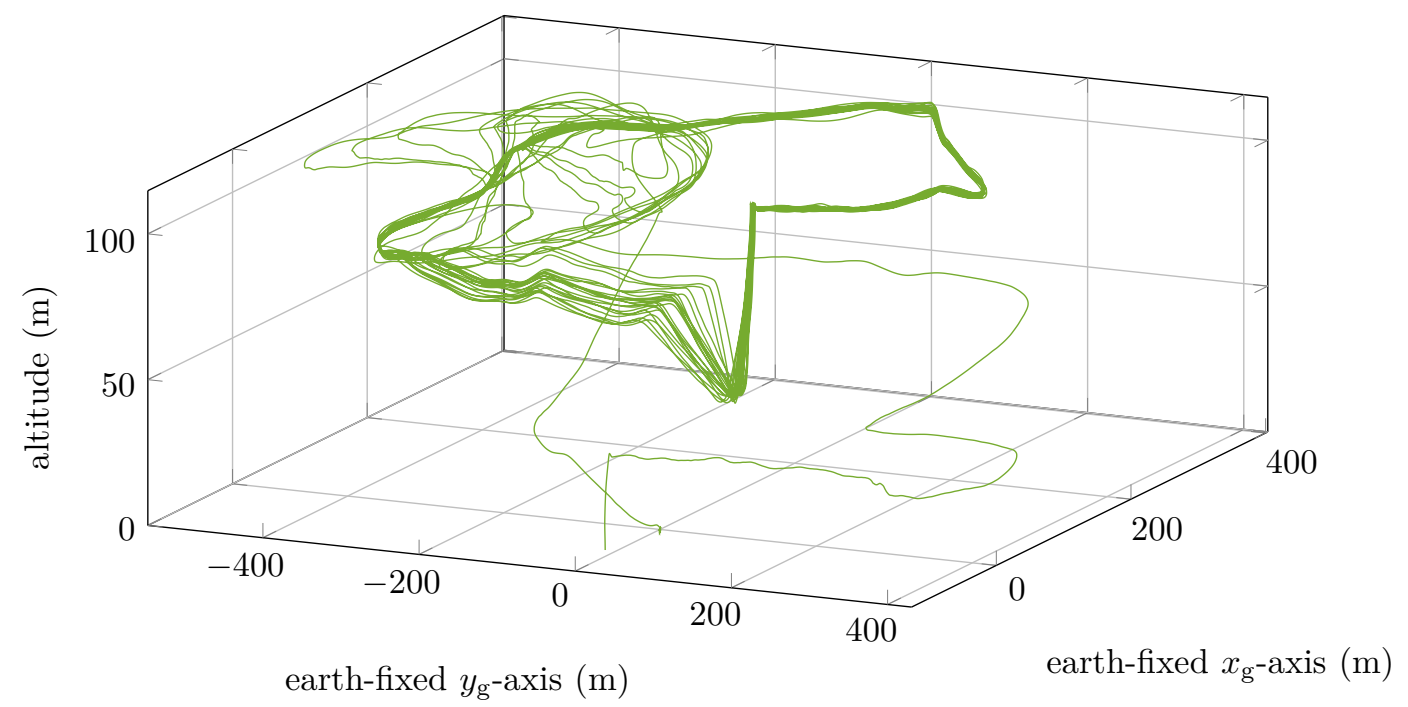

Fig. 2: Flight-path of Cumulus One with 22 aborted deep stall manoeuvres. The earth-fixed axis system is originated in the initial position of the drone when arming.

\section{The Cumulus One}

This section introduces the Cumulus drone (Fig. 1), its models, and the data from flight experiment which is to be used in this study. The Cumulus UA is manufactured and sold by the Danish company SkyWatch for autonomous agricultural mapping, surveillance, and reconnaissance.

\section{A. Unmanned aircraft}

Fig. 1a shows a digital rendering of the assembled Cumulus UA. It has a wingspan of $1.65 \mathrm{~m}$, a typical mass of $1.7 \mathrm{~kg}$, and an endurance of $150 \mathrm{~min}$. With a payload capability of up to $500 \mathrm{~g}$ it offers flexibility in sensor combinations. Cumulus One has a range of $10 \mathrm{~km}$ for beyond visual line of sight operations, a cruise speed of $16 \mathrm{~m} / \mathrm{s}$, is just over one metre long, and it is handlaunched. [19]

Cumulus is designed for deep stall landing. In Fig. 1b a special shock-absorbing foam can be seen at the belly of the aircraft. This enables repeated deep stall landing without damaging the drone. Also shown are the propellers that fold back when not spinning [20]. This improves performance during deep stall where no thrust is necessary and the flight angle is different from usual, levelled flight. In order to be able to perform the deep stall, Cumulus is equipped with an elevator which allows for large negative deflections. This both serves to initiate the deep stall and to maintain a desired attitude during the descent.

The Cumulus UA is fully automated once it is launched. Its onboard instrumentation includes inertial measurement units (IMUs) with accelerometer, gyroscope, and magnetometer triads as well as barometric sensors and a GPS unit.

\section{B. Experimental flights}

Fig. 2 shows the flight-path of an experiment containing 22 aborted deep stall manoeuvres and one deep stall landing. The cruising altitude was $100 \mathrm{~m}$, at which the Cumulus passes a series of predefined way points prior to each deep stall. Cumulus descends to an altitude of $50 \mathrm{~m}$ at which point the deep stall manoeuvre is aborted and a predefined recovery strategy is executed. The Cumulus drone then returns to cruising altitude to perform the next aborted deep stall manoeuvre. Some deviations in the flight part are seen in Fig. 2. They are the result of the operator taking manual control for adjusting controller settings. When returned to autopilot Cumulus resumes its original mission. After the 22 manoeuvres it descents to an altitude of $30 \mathrm{~m}$ and performs a deep stall landing. Tab. I shows the parameters of the particular Cumulus One used during the experiment.

TABLE I: Parameters for the experiment.

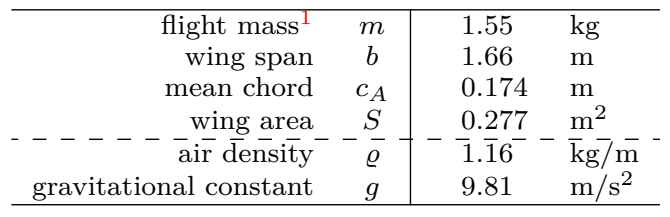

\section{Virtual instrumentation $\&$ Flight logs}

The on-board software provides extensive logs of both sensor data and actuator commands. Further navigation variables are estimated by a 22-state Extended Kalman filter (EKF) implementation. The EKF states include the global position, the flight-path velocity $\mathbf{V}_{K}$, the

\footnotetext{
${ }^{1}$ Total mass at take-off, including payload
} 


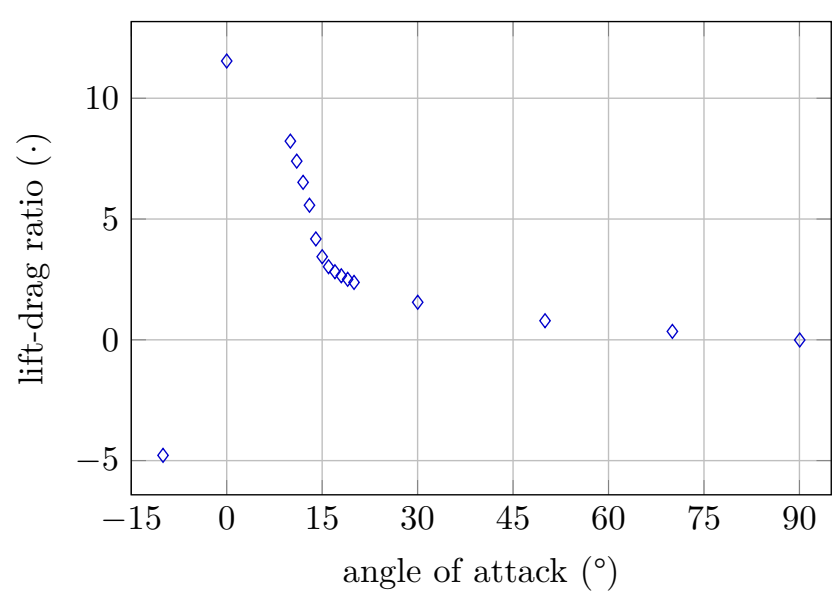

Fig. 3: Lift-drag ratio $C_{\mathrm{L}} / C_{\mathrm{D}}$ by $\mathrm{CFD}$ for zero elevator.

horizontal wind velocity $\mathbf{V}_{\mathrm{W}}$, and the attitude. The airpath velocity is then given as

$$
\mathbf{V}_{\mathrm{A}}=\mathbf{V}_{\mathrm{K}}-\mathbf{V}_{\mathrm{W}},
$$

As common, we have air speed $V_{\mathrm{A}}$ as norm of $\mathbf{V}_{\mathrm{A}}$ and the angle of attack $\alpha$ and angle of side-slip $\beta$ are calculated as in [21]. The on-board accelerometers are positioned close to the center of gravity of the plane and the measurements are further post-processed by a lowpass filter.

The aerodynamic coefficients of the Cumulus One body have been simulated using Computational Fluid Dynamics (CFD). The data, exemplary in Fig. 3, have been used for verification of the in-flight identification.

\section{Preliminaries}

In this paper, we will refer to the axis systems of the international standard [21] (Fig. 4): the body axis system aligned with fuselage; the air-path axis system aligned with the air-path velocity vector $\mathbf{V}_{\mathrm{A}}$; the flight-path axis system aligned with the flight-path velocity vector $\mathbf{V}_{\mathrm{K}}$; and the normal earth-fixed axis system. Variables with respect to this axis systems are denoted by indices ${ }_{\mathrm{f}}$, a, $\mathrm{k}$, and $\mathrm{g}_{\mathrm{g}}$, respectively. The rotation matrix from $\mathbf{x}_{\mathrm{s}}$ to $\mathbf{x}_{\mathrm{t}}$ is given by $\boldsymbol{\Omega}_{\mathrm{ts}}$ with $\mathbf{x}_{\mathrm{t}}=\boldsymbol{\Omega}_{\mathrm{ts}} \mathbf{x}_{\mathrm{s}}$. We denote the vectors

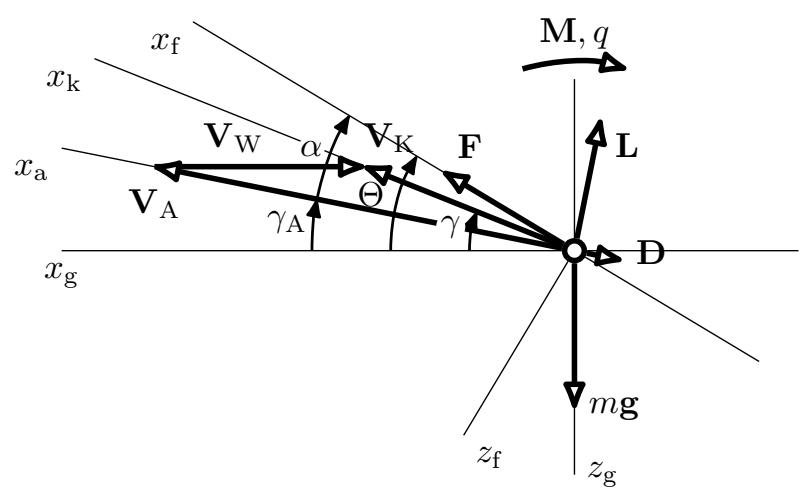

Fig. 4: Longitudinal axes with angles and vectors. of forces and accelerations by $\mathbf{R}=\left[\begin{array}{lll}X & Y & Z\end{array}\right]^{T}$ and $\mathbf{a}=\left[\begin{array}{lll}a_{x} & a_{y} & a_{z}\end{array}\right]^{T}$, respectively. Here, the forces are marked by superscript ${ }^{\mathrm{A}}$ (aerodynamic), ${ }^{\mathrm{F}}$ (thrust), or G (weight).

Accelerations are measured in the inertial reference frame of the accelerometer, thus we have the measured accelerations $\tilde{\mathbf{a}}_{\mathrm{f}}$ in the body axis system to

$$
\tilde{\mathbf{a}}_{\mathrm{f}}=\mathbf{a}_{\mathrm{f}}-\boldsymbol{\Omega}_{\mathrm{fg}}\left[\begin{array}{l}
0 \\
0 \\
g
\end{array}\right]+\Delta \mathbf{a}_{\mathrm{f}},
$$

where $\mathbf{a}_{\mathrm{f}}$ is the actual acceleration of the body and $\Delta \mathbf{a}_{\mathrm{f}}$ are measurement errors.

\section{IN-FLIGHT FORCE ESTIMATION}

The accelerations of the aircraft are the result of the sum of acting forces,

$$
\sum_{i} \mathbf{R}_{\mathrm{f}}^{i}=m \mathbf{a}_{\mathrm{f}}
$$

where $m$ is the mass of the vehicle. During flight, the aerodynamic forces, thrust, and weight act on the aircraft:

$$
m \mathbf{a}_{\mathrm{f}}=\mathbf{R}_{\mathrm{f}}^{\mathrm{A}}+\mathbf{R}_{\mathrm{f}}^{\mathrm{F}}+\mathbf{R}_{\mathrm{f}}^{\mathrm{G}}
$$

or, in the aerodynamic axis system,

$$
m \boldsymbol{\Omega}_{\mathrm{af}} \mathbf{a}_{\mathrm{f}}=\left[\begin{array}{c}
-D \\
Q \\
-L
\end{array}\right]+\boldsymbol{\Omega}_{\mathrm{af}}\left[\begin{array}{c}
F \\
0 \\
0
\end{array}\right]+\boldsymbol{\Omega}_{\mathrm{ag}}\left[\begin{array}{l}
0 \\
0 \\
g
\end{array}\right],
$$

where $F$ is the thrust; and aerodynamic lift, drag, and side-force are given by the aerodynamic coefficients as

$$
\begin{aligned}
L & =\frac{1}{2} \varrho V_{\mathrm{A}}^{2} S C_{\mathrm{L}} ; \\
D & =\frac{1}{2} \varrho V_{\mathrm{A}}^{2} S C_{\mathrm{D}} ; \\
Q & =\frac{1}{2} \varrho V_{\mathrm{A}}^{2} S C_{\mathrm{Q}} ;
\end{aligned}
$$

with air density $\varrho$ and reference area $S$. Re-ordering (5) and applying (2), we get

$$
m \tilde{\mathbf{a}}_{\mathrm{a}}=\mathbf{R}_{\mathrm{a}}^{\mathrm{A}}+\boldsymbol{\Omega}_{\mathrm{af}} \mathbf{R}_{\mathrm{f}}^{\mathrm{F}} .
$$

In the flight shown in Fig. 2 the autopilot is applying a throttle command in almost the entire flight. Initially we only know the thrust $F$ during the aborted deep stalls since the throttle command is zero. We can then calculate the aerodynamic coefficients during the descents by solving (6)-(9) for $C_{\mathrm{L}}, C_{\mathrm{D}}$, and $C_{\mathrm{Q}}$. Thus calculated coefficients for pitch rate $-0.1 \% \leq q \leq 0.1 \%$ are exemplary shown in Fig. 5. The mean elevator deflection here has been $\eta \approx-45^{\circ}$.

However, if the thrust is unknown and different from zero, as for the full envelope range of the flight experiments here, estimation of the aerodynamic coefficients is difficult. To overcome this challenge, we will derive a thrust model based on the force estimation at low angles of attack and extend it to the full envelope. 


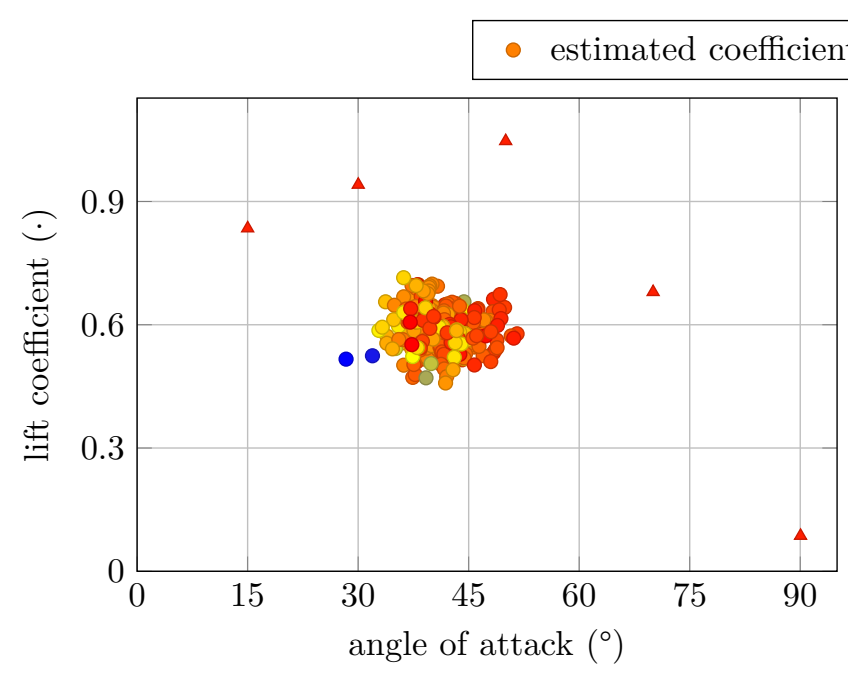

(a) Lift coefficient $C_{\mathrm{L}}$.

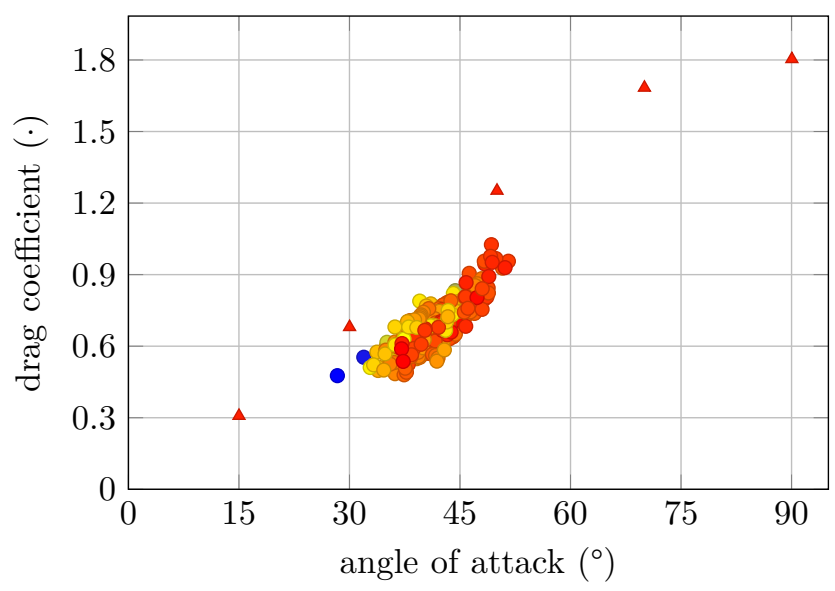

(b) Drag coefficient $C_{\mathrm{D}}$.

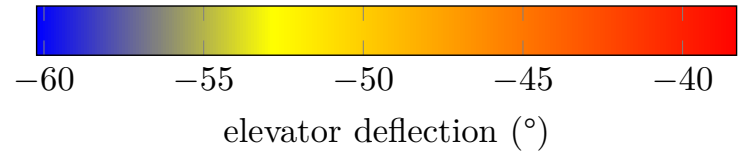

Fig. 5: Zero-thrust force estimation compared to CFD.

\section{Parameter Identification}

In this section we identify the parameters of the thrust model for low angles of attack aerodynamic coefficients based on the estimation of forces encountered during flight.

\section{A. Thrust model}

The thrust generation of a propeller depends on its geometry, the rotation speed, the air speed, and the incidence angle [22]. We then have

$$
F=\varrho n^{2} D^{4} C_{\mathrm{F}}
$$

with the revolutions per second $n$, the diameter $D$ and thrust coefficient $C_{\mathrm{F}}$. We also introduce the advanced ratio

$$
J=\frac{V_{\mathrm{A}}}{n D} .
$$

In [23], a linear relation between thrust coefficient, revolutions per second, and advanced ratio has been proposed for a normal range of air speeds:

$$
C_{\mathrm{F}}=C_{\mathrm{F} 0}+C_{\mathrm{F} J} J+C_{\mathrm{F} n} n
$$

with the coefficients $C_{\mathrm{F} 0}, C_{\mathrm{F} J}, C_{\mathrm{F} n}$. This assumption was then successfully evaluated in wind-tunnel experiments for different propeller models and types. We further assume a linear relation between motor rotation speed and throttle command to the speed controller, $n \propto \tau$, and obtain

$$
\begin{aligned}
F & =\varrho n^{2} D^{4}\left(C_{\mathrm{F} 0}+C_{\mathrm{F} J} \frac{V_{\mathrm{A}}}{n D}+C_{\mathrm{F} n} n\right) \\
& =C_{\mathrm{F} 1} \tau \cdot V_{\mathrm{A}}+C_{\mathrm{F} 2} \tau^{2}+C_{\mathrm{F} 3} \tau^{3}
\end{aligned}
$$

for suitable coefficients $C_{\mathrm{F} 1}, C_{\mathrm{F} 2}, C_{\mathrm{F} 3}$.

\section{B. Modeling for low angles of attack}

For low angles of attack, we can use the well-known representation of the lift and drag coefficients being linear (squared) in the angle of attack:

$$
\left\{\begin{array}{l}
C_{\mathrm{L}}=C_{\mathrm{L} 0}+C_{\mathrm{L} \alpha} \alpha \\
C_{\mathrm{D}}=C_{\mathrm{D} 0}+C_{\mathrm{D} \alpha} \alpha^{2}
\end{array}\right.
$$

The forces along the aerodynamic $x_{\mathrm{a}}$ and $z_{\mathrm{a}}$ axes are then derived from (9) to

$$
\begin{aligned}
m\left[\begin{array}{c}
\tilde{a}_{x} \\
0 \\
\tilde{a}_{z}
\end{array}\right]_{\mathrm{a}}= & -\frac{1}{2} \varrho V_{\mathrm{A}}^{2} S\left[\begin{array}{c}
C_{\mathrm{D} 0}+C_{\mathrm{D} \alpha} \alpha^{2} \\
0 \\
C_{\mathrm{L} 0}+C_{\mathrm{L} \alpha} \alpha
\end{array}\right] \\
& +\boldsymbol{\Omega}_{\mathrm{af}}\left[\begin{array}{c}
C_{\mathrm{F} 1} \tau \cdot V_{\mathrm{A}}+C_{\mathrm{F} 2} \tau^{2}+C_{\mathrm{F} 3} \tau^{3} \\
0 \\
0
\end{array}\right],
\end{aligned}
$$

by substitution of (14) and (15).

\section{Optimal parameters}

As the assumption of (15) is only valid for low angles of attack, we choose the limits to be $\pm 10^{\circ}$. If we evaluate (16) in the data points $\tilde{a}_{x i}, \tilde{a}_{z i}, V_{\mathrm{A} i}, \alpha_{i}, \beta_{i}, \tau_{i}$ from the flight logs with $i \in I_{\alpha} \Longleftrightarrow-10^{\circ} \leq \alpha_{i} \leq 10^{\circ}$ and $m, \varrho, S$ from Table I, we find optimal parameters for (14) and (15) by linear least-square (LSQ) optimization:

$$
\mathbf{q}_{\mathrm{opt}}=\arg \min _{\mathbf{q}}\|\mathbf{C q}-\mathbf{d}\|_{2}^{2} .
$$

Here, the solution vector is given as

$$
\mathbf{q}_{\mathrm{opt}}=\left[\begin{array}{lllllll}
C_{\mathrm{F} 1} & C_{\mathrm{F} 2} & C_{\mathrm{F} 3} & C_{\mathrm{L} 0} & C_{\mathrm{L} \alpha} & C_{\mathrm{D} 0} & C_{\mathrm{D} \alpha}
\end{array}\right]^{T}
$$




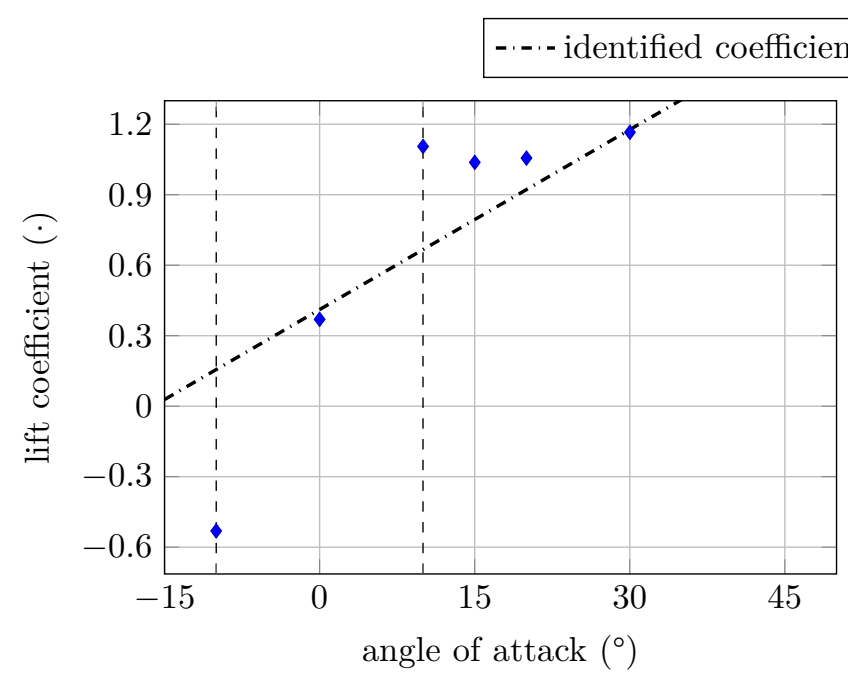

(a) Lift coefficient $C_{\mathrm{L}}$.
- $\operatorname{CFD}\left(\eta=-2^{\circ}\right)$

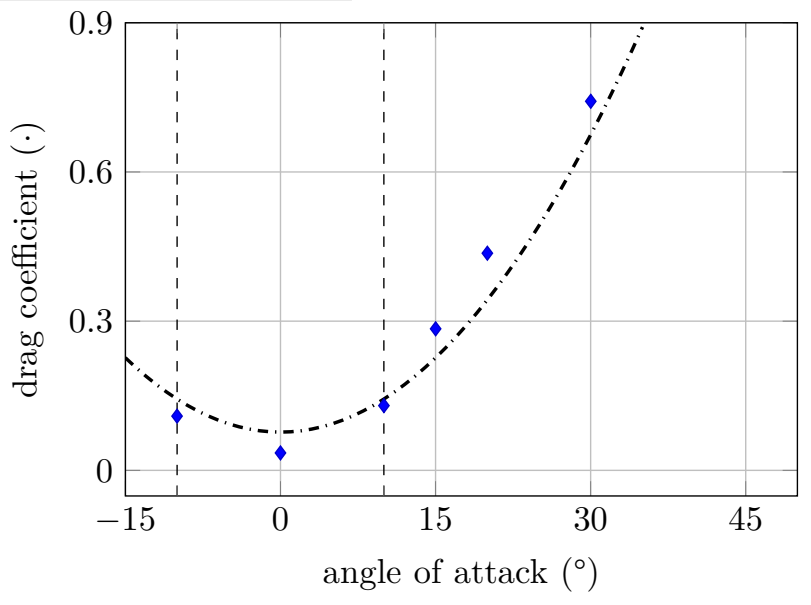

(b) Drag coefficient $C_{\mathrm{D}}$.

Fig. 6: Identified aerodynamic coefficients for low angles of attack $\left(-10^{\circ} \leq \alpha \leq 10^{\circ}\right)$ compared to CFD.

and we have the objective matrix

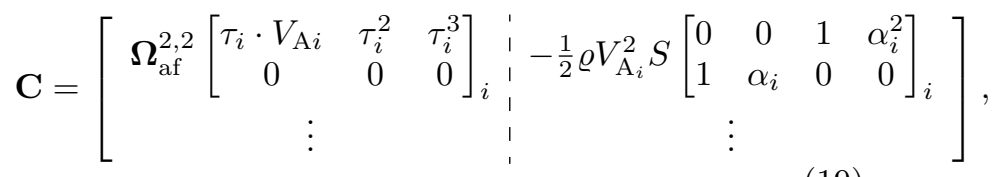

The optimal parameters are identified using the lsqlin function of the MOSEK software [24] and can be found in the Appendix. The identified aerodynamic coefficients for low angles of attack and the identified thrust model are shown in Fig. 6 and 7, respectively. where $\boldsymbol{\Omega}_{\mathrm{af}}^{2,2}$ denotes the submatrix of $\boldsymbol{\Omega}_{\mathrm{af}}$ obtained by deleting each the $2^{\text {nd }}$ row and column, and the target vector

$$
\mathbf{d}=\left[\begin{array}{c}
m\left[\begin{array}{c}
\tilde{a}_{x i} \\
\tilde{a}_{z i}
\end{array}\right]_{i} \\
\vdots
\end{array}\right]
$$

for all $i \in I_{\alpha}$.

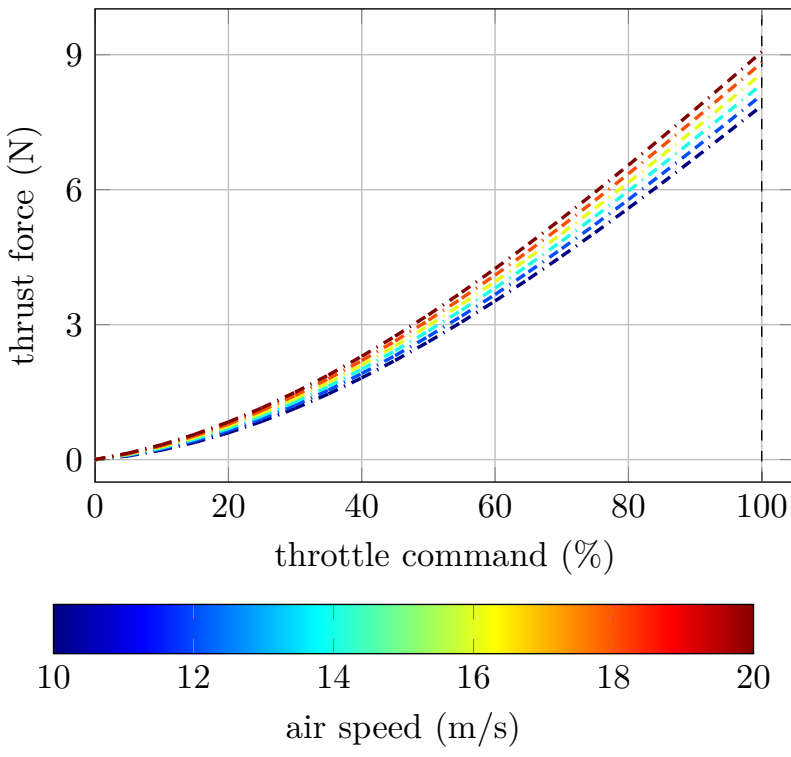

Fig. 7: Identified thrust model $F\left(\tau, V_{\mathrm{A}}\right)$.

\section{Discussion}

In order to identify the parameters of the thrust model based on the low angle of attack models of the aerodynamic coefficients, we have silently met some assumptions which do not trivially hold. We shall present these requirements now and argue their validity for the given flight data. Subsequently, we will discuss the results of the identification and the possible influence of parametric uncertainties.

First, a requirement for a multi-variable fitting of parameters is stochastic independence of the respective variables, here the angle of attack $\alpha$ and throttle $\tau$. The consequences of stochastically dependent variables can be well observed in Fig. 8: the left figure shows a dimensional deficit compared to the right; i.e., the regression surface diminishes to a curve and a parametric optimizer cannot distinguish between the effects of $X$
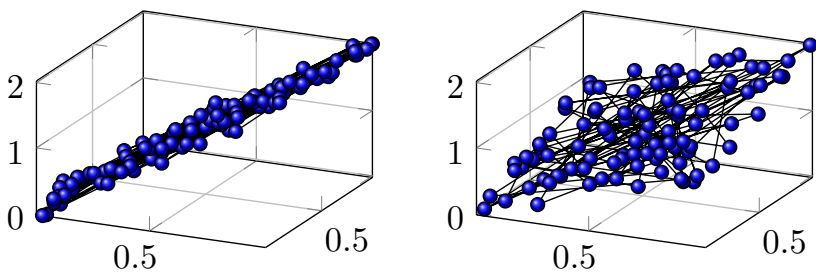

Dependent variables $X \sim Y$. Independent variables $X, Y$.

Fig. 8: Effects of stochastically dependent variables on the example $f:(X, Y) \mapsto X+Y$. 
and $Y$ to $f$. The linear dependency of stochastic variables can be measured by the Pearson correlation coefficient $\rho(X, Y) \in[-1 ; 1]$; values closer to +1 indicate dependency of $X$ and $Y$. For the variables of the thrust model identification we have

$$
\begin{aligned}
\rho\left(\alpha_{i}, \tau_{i}\right) & =-0.32 ; \\
\rho\left(\alpha_{i}, V_{\mathrm{A}_{i}}\right) & =-0.29 ;
\end{aligned}
$$

but

$$
\rho\left(\tau_{i}, V_{\mathrm{A}_{i}}\right)=0.41,
$$

indicating a correlation between throttle command and air speed which has been neglected here.

Moreover, when modeling the lift and drag coefficients we have ignored the effects of further variables such as elevator, side-slip, or pitch rate. Table II shows the distribution of those variables for low angles of attack. While they clearly contribute to lift and drag, the LSQ problem structure averages their influence. Hence, the identified parameters are valid for side-slip and pitch rate close to zero, and an elevator deflection of $\eta \approx-1.75^{\circ}$.

TABLE II: Mean values $(\mu)$ and standard deviations $(\sigma)$ of further variables during low angle of attack flight.

\begin{tabular}{rl|rll} 
Variable & & \multicolumn{1}{|c}{$\mu$} & \multicolumn{1}{c}{$\sigma$} & \\
\hline side-slip & $\beta$ & 0.10 & 2.86 & $\circ$ \\
elevator & $\eta$ & -1.75 & 1.47 & $\circ$ \\
pitch rate & $q$ & 0.10 & 2.86 & $\circ / \mathrm{s}$ \\
\hline
\end{tabular}

As shown in Fig. 6, the identified drag coefficients track the CFD data well for low angle of attack and neighbouring points; the identified slope of the lift coefficients clearly underestimates the CFD data. This offset, also apparent in unthrottled flight (Fig. 5), is likely caused by inaccurate estimations of wind and air speed as well as biased accelerometer measurements. The thrust model (Fig. 7) shows small alterations at different air speeds and the resulting thrust increases with the air speed, opposite to the expectation [22]. This can be deduced to the stochastic dependency of the throttle and air speed values at low angles of attack. For small angles of attack and inclination angles, the applied forces are nearly perpendicular (Fig. 4) with lift opposing weight, thrust opposing drag. That is, the lift model is rather affected by inaccuracies in the weight while an accurate drag model supports the thrust model estimation.

The fitted results are further subject to parametric uncertainties; in particular, flight mass and air density will vary from flight to flight. While variations of the air density affects the identified lift and drag coefficients, uncertainties of the mass have an interesting effect: since

$$
\min _{\mathbf{q}}\|\mathbf{C q}-\mathbf{d}\|_{2}^{2}=m \cdot \min _{\tilde{\mathbf{q}}}\|\mathbf{C} \tilde{\mathbf{q}}-\mathbf{d} / m\|_{2}^{2}
$$

with $\tilde{\mathbf{q}}=\mathbf{q} / m$, the parameters can be identified without knowledge of $m$ first. The parameters of lift, drag, and thrust model are then obtained by $\mathbf{q}=m \tilde{\mathbf{q}}$; that is, the identified model is scaled by the aircraft mass.

\section{Aerodynamics in Deep-Stall Flight}

In this section the derived thrust model is used to calculate the instantaneous drag and lift coefficients. With the coefficients $C_{\mathrm{F} 1}, C_{\mathrm{F} 2}$, and $C_{\mathrm{F} 3}$ determined as the solution to (17), equations (6), (7), and (9) are solved for each data point during the flight. Figures 9, 10, and 11 show the calculated drag coefficient through the entire flight as a function of angle of attack $\alpha$, the pitch rate $q$, and the elevator deflection $\eta$. Fig. 10 shows the $\alpha-q$ plane and Fig. 11 shows the $q-\eta$ plane.

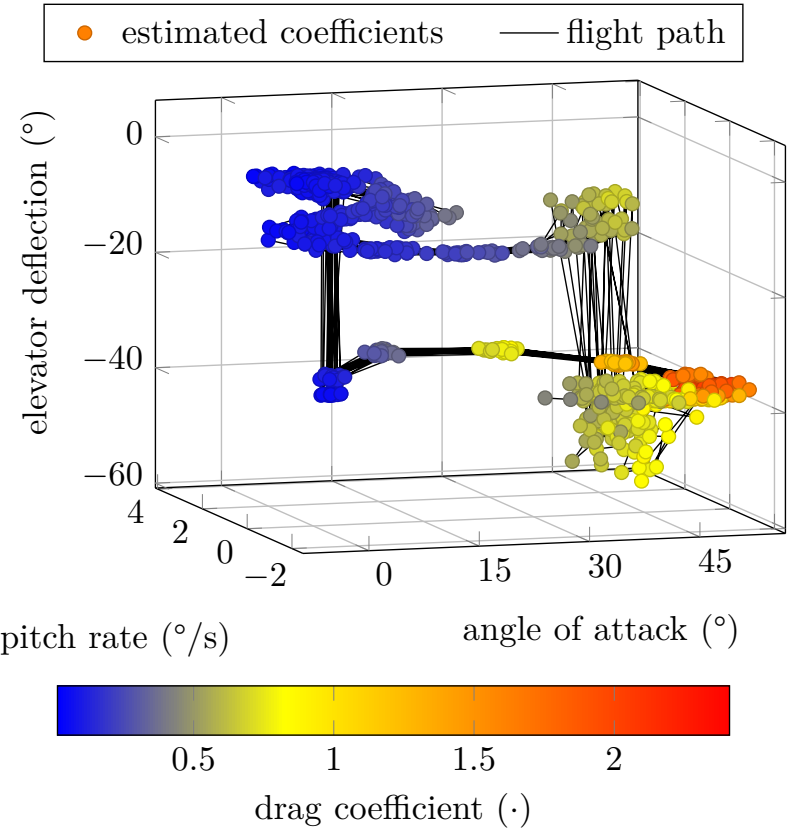

Fig. 9: Drag coefficient plotted as a function of angle of attack $\alpha$, pitch rate $q$, and elevator deflection $\eta$. The transition from normal flight with $C_{\mathrm{D}} \approx 0.3$ to deep stall descent with $C_{\mathrm{D}} \approx 1$ is clearly visible.

The distinct nature of initiating and aborting a deep stall is clearly visible in these figures. In regular, leveled flight, the three variables are in the intervals $\alpha \in$ $\left[-10^{\circ} ; 10^{\circ}\right], q \in\left[-0.50^{\circ} / \mathrm{s} ; 0.70^{\circ} / \mathrm{s}\right]$, and $\eta \in\left[-5^{\circ} ; 1^{\circ}\right]$. On Fig. 10 this is seen around the origin at the high concentration of data points. On Fig. 11 this is seen as the elliptic shape with high concentration of data points close to the origin. On Fig. 9 it is seen as the top left corner of the points. At some point, the mission requires the Cumulus drone to do a deep stall, usually because the mission is over. This is initiated by a step in elevator deflection. The controllers no longer attempt to maintain a desired attitude or flight angle, but simply sets the elevator deflection to $\eta \approx-38^{\circ}$. This is best seen in Fig. 9 where the data points drop to approximately $[0,0,-38]$ and then transitions through a half circle with a top point of $[20,4,-38]$ and an end point of $[50,0,-38]$. This half circle is best seen in Figs. 9 and 10. This transition is the initiation of the deep stall where the forward velocity is almost completely converted to a small increase in 
altitude. Compared to Fig. 2 it is the small bump before the descents. The change in color clearly shows the change in drag coefficient throughout this manoeuvre. After the Cumulus drone has lost its forward velocity it starts to descent. At this point the elevator is again free to move and the controllers attempts to maintain a pitch of $\theta=-10^{\circ}$ throughout the descent. This is best seen in Fig. 11 where the data points encircle the points $[\alpha, 0,-46]$ with $\alpha \in\left[32^{\circ} ; 50^{\circ}\right]$ depending on the particular descent.

For a regular landing the Cumulus drone would maintain a pitch of $\theta=-10^{\circ}$ until impact. In the aborted deep stall manoeuvres considered in this paper the descents are aborted once the altitude falls below 50 meters. At this point the Cumulus initiates a predefined abort scheme. The elevator is stepped to a constant value of $\eta \approx-11^{\circ}$. This is seen on Fig. 9 where the data points jumps to around $[40,0,-11]$ and then transitions through a second half circle with a bottom point of $[20,-2,-11]$ and an end point of $[0,0,-11]$. This half circle is best seen on Fig. 10. Stepping the elevator back down towards zero makes the drone pitch forward as observed on the pitch rate. Thanks to gravity the air speed vector is still dominated by its vertical component, but since the drone pitches forward the angle of attack diminishes. This enables for the Cumulus to return to normal fight mode and regain control. On Figs. 9 and 10 the pitch rate can be seen to become positive again, and shortly after the angle of attack is around $\alpha \approx 15^{\circ}$. At this point the elevator is again free to be controlled. Before returning to regular, levelled flight the angle of attack remains around $\alpha \approx 10^{\circ}$ where the elevator deflection is $\eta \in\left[-11^{\circ} ;-5^{\circ}\right]$. During this time Cumulus changes its altitude from $35 \mathrm{~m}$ to $100 \mathrm{~m}$ after which it is back in the usual operation mode where the data points are dense as described in the beginning of this section.

A similar graph has been obtained for the lift coefficient. It exhibits the same tendencies as observed in Fig. 9, and is not presented here.

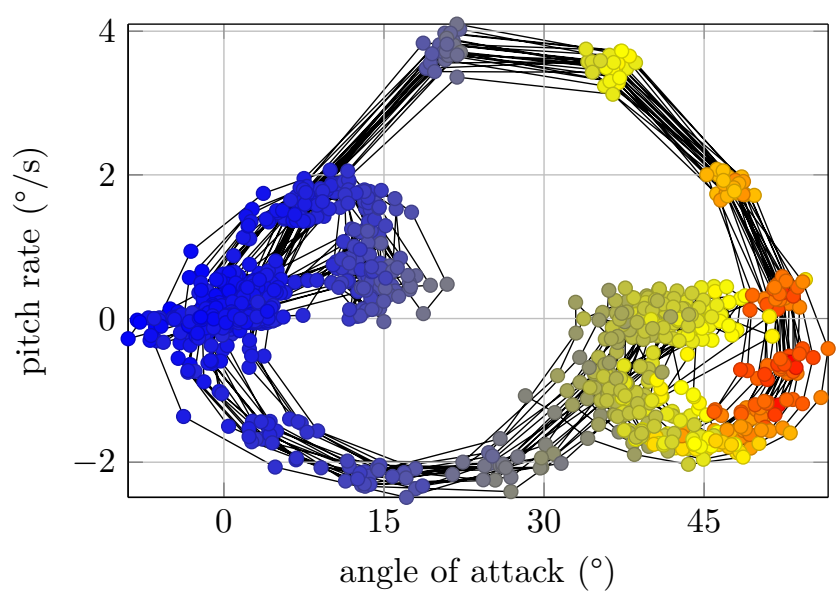

Fig. 10: Projection of Fig. 9 into the $\alpha-q$ plane.

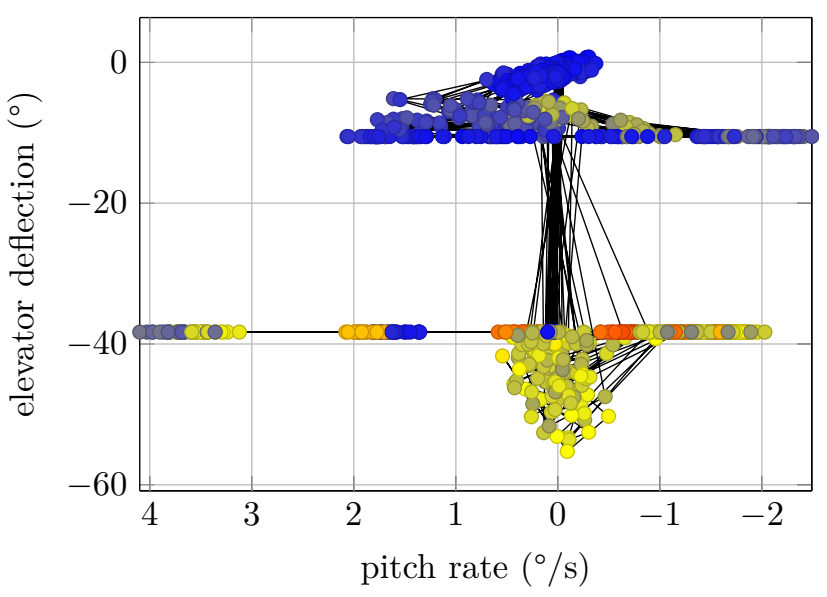

Fig. 11: Projection of Fig. 9 into the $q-\eta$ plane.

\section{Conclusion}

In-flight force estimation provides aerodynamic details of an aerial vehicle but requires knowledge of the applied thrust force. In this paper, we have obtained a thrust model using experiment data of the Cumulus One UA in regular and deep-stalled flight. The identification method is based on a linearity assumption of thrust, justified by previous wind-tunnel studies, as well as of lift and drag at low angles of attack. Parameters are hence found by linear least-square optimization. With the identified thrust model, we have determined lift and drag coefficients in the full envelope, including high angles of attack, elevator deflections, and pitch rates. The calculations show good general agreement with the available CFD, given the present uncertainties of the estimated air speed and angle of attack as well as the measured accelerations. Future work includes air measurements by pitot sensors and improved accelerometer data.

\section{APPENDIX}

The transformation matrix used in this paper are given by [25]

$$
\boldsymbol{\Omega}_{\mathrm{af}}=\boldsymbol{\Omega}_{\mathrm{fa}}^{T}=\left[\begin{array}{ccc}
\cos \alpha \cos \beta & \sin \beta & \sin \alpha \cos \beta \\
-\cos \alpha \sin \beta & \cos \beta & -\sin \alpha \sin \beta \\
-\sin \alpha & 0 & \cos \alpha
\end{array}\right] .
$$

The optimal parameters found in Sec. V-C are given as

$$
\begin{array}{llrl}
C_{\mathrm{F} 1} & =0.1544 & & C_{\mathrm{F} 2}=12.25 \\
C_{\mathrm{F} 3} & =-3.642 & & \\
C_{\mathrm{L} 0} & =0.5005 & & C_{\mathrm{L} \alpha}=1.781 \\
C_{\mathrm{D} 0} & =0.09362 & & C_{\mathrm{D} \alpha}=2.656 .
\end{array}
$$

\section{ACKNOWLEDGEMENT}

The authors wish to thank Vamsi Krishna Dasam for his invaluable work providing the CFD simulations; Murat Bronz for his advice on propeller thrust models; and Donatas Poteliunas for test flights and flight logs. 


\section{REFERENCES}

[1] A. Parsch, "AeroVironment RQ-11 Raven," Directory of U.S. Military Rockets and Missiles, Tech. Rep., 2006. [Online]. Available: http://www. designation-systems.net/dusrm/app2/q-11.html

[2] - "AeroVironment RQ-14 Dragon Eye / Swift," Directory of U.S. Military Rockets and Missiles, Tech. Rep., 2007. [Online]. Available: http://www. designation-systems.net/dusrm/app2/q-14.html

[3] W. Pointner, G. Kotsis, P. Langthaler, and M. Naderhirn, "Using formal methods to verify safe deep stall landing of a MAV," in 30th AIAA/IEEE Digital Avionics Systems Conference, Seattle, USWA, oct 2011, pp. 1-10.

[4] S. H. Mathisen, T. I. Fossen, and T. A. Johansen, "Non-linear model predictive control for guidance of a fixed-wing UAV in precision deep stall landing," in 2015 International Conference on Unmanned Aircraft Systems, Denver, US-CO, jun 2015, pp. 356-365.

[5] N. K. Ure and G. Inalhan, "Autonomous control of unmanned combat air vehicles: Design of a multimodal control and flight planning framework for agile maneuvering," IEEE Control Systems Magazine, vol. 32, no. 5, pp. 74-95, 2012.

[6] E. Bulka and M. Nahon, "Autonomous control of agile fixed-wing UAVs performing aerobatic maneuvers," in 2017 International Conference on Unmanned Aircraft Systems, Miami, US-FL, jun 2017, pp. 104-113.

[7] T. Cunis, L. Burlion, and J.-P. Condomines, "Nonlinear Analysis and Control Proposal for In-flight Loss-of-control," in 20th IFAC World Congress, preprints, Toulouse, FR, jul 2017, Publication.

[8] M. S. Selig, "Modeling Full-Envelope Aerodynamics of Small UAVs in Realtime," in AIAA Atmospheric Flight Mechanics Conference, Toronto, CA, aug 2010, pp. 1-35.

[9] W. Khan and M. Nahon, "Modeling dynamics of agile fixed-wing UAVs for real-time applications," in 2016 International Conference on Unmanned Aircraft Systems, Arlington, US-VA, jun 2016, pp. 1303-1312.

[10] B. F. Mettler, "Extracting Micro Air Vehicles Aerodynamic Forces and Coefficients in Free Flight Using Visual Motion Tracking Techniques," Experiments in Fluids, vol. 49, pp. 557-569, 2010.

[11] A. H. Rao, D. V. Uhlig, and M. S. Selig, "Glide and Powered Flight Characteristics of Micro Air Vehicles from Experimental Measurements," in AIAA $A p$ plied Aerodynamics Conference, New Orleans, USLA, jun 2012.

[12] J. Moore, R. Cory, and R. Tedrake, "Robust poststall perching with a simple fixed-wing glider using LQR-Trees," Bioinspiration and Biomimetics, vol. 9, no. 2, 2014.
[13] R. W. Deters, G. K. Ananda, and M. S. Selig, "Reynolds Number Effects on the Performance of Small-Scale Propellers," 32nd AIAA Applied Aerodynamics Conference, pp. 1-43, jun 2014.

[14] M. Silvestre, J. Morgado, P. Alves, P. Santos, P. Gamboa, and J. Páscoa, "Propeller Performance Data at Low Reynolds Numbers," International Journal of Mechanics, vol. 9, pp. 154-166, 2015.

[15] M. H. McCrink and J. W. Gregory, "Blade Element Momentum Modeling of Low-Reynolds Electric Propulsion Systems," Journal of Aircraft, vol. 54, no. 1, pp. 163-176, 2017.

[16] L. Pivano, T. A. Johansen, and Ø. N. Smogeli, "A four-quadrant thrust estimation scheme for marine propellers: Theory and experiments," IEEE Transactions on Control Systems Technology, vol. 17, no. 1, pp. 215-226, 2009.

[17] M. Henriksson, T. Grönstedt, and C. Breitholtz, "Model-based on-board turbofan thrust estimation," Control Engineering Practice, vol. 19, no. 6, pp. 602-610, 2011.

[18] C. L. Lawson and R. J. Hanson, Solving Least Squares Problems, ser. Classics in Applied Mathematics. Society for Industrial and Applied Mathematics, 1995.

[19] "The Cumulus v1 UAV," 2017. [Online]. Available: http://sky-watch.com/products/cumulus/

[20] "CAM Carbon-Blades 14.0x8.0in," aero-naut Modellbau GmbH \& Co KG, Reutlingen, DE, Technical report, 2018.

[21] ISO 1151-1, Flight dynamics - Concepts, quantities and symbols - Part 1: Aircraft motion relative to the air, 4th ed. Genève, CH: International Organization for Standarization, 1988.

[22] W. F. Phillips, Mechanics of Flight, 2nd ed. Hoboken, US-NJ: John Wiley \& Sons, 2010.

[23] G. Hattenberger, A. Drouin, and M. Bronz, "Electric Propulsion System Characterization through Experiments," in 8th Internatioal Micro Air Vehicle Conference and Competition, Beijing, CN, 2016.

[24] "MOSEK Optimization Software," 2018. [Online]. Available: https://www.mosek.com/products/ mosek/

[25] R. Brockhaus, W. Alles, and R. Luckner, Flugregelung, 3rd ed. Berlin, DE: Springer, 2011. 